\title{
1 The composition of the microbiota in the \\ 2 full-term fetal gut and amniotic fluid: a \\ 3 bovine caesarean section study \\ 4
}

5 Aleksi Husso*, Leen Lietaer*, Tiina Pessa-Morikawa, Thomas Grönthal, Jan

6 Govaere, Ann Van Soom, Antti Iivanainen**, Geert Opsomer**, Mikael Niku**

$7 \quad$ *First authors contributed equally

$8 \quad * *$ supervised the study

9 Corresponding author: Mikael Niku, mikael.niku@helsinki.fi

10

\section{Abstract}

The fetal development of the intestinal immune system is stimulated by the maternal microbiota, but it is still unclear whether viable bacteria exist in the healthy fetus. Analysis of such low microbial biomass environments are challenging due to contamination issues. The aims of the current study were to assess the bacterial load and characterize the bacterial composition of the amniotic fluid and meconium of full-term calves, leading to a better knowledge of prenatal bacterial seeding of the fetal intestine. Amniotic fluid and rectal meconium samples were collected during and immediately after elective caesarean section, performed in 25 Belgian Blue cow-calf couples. The samples were analyzed by qPCR, bacterial culture using GAM agar and 16S rRNA gene amplicon sequencing. To minimize the effects of contaminants, we included multiple technical controls and stringently filtered the 16S rRNA gene sequencing data to exclude putative contaminant sequences. The meconium samples contained a significantly higher amount of bacterial DNA than the negative controls and 5 of 24 samples contained culturable bacteria. In the amniotic fluid, the amount of bacterial DNA was not significantly different from the negative controls and all samples were culture negative. 
Bacterial sequences were identified in both sample types and were primarily of phyla

Proteobacteria, Firmicutes, Bacteroidetes and Actinobacteria, with some individual variation.

We conclude that most calves encounter in utero maternal-fetal transmission of bacterial DNA, but the amount of bacterial DNA is low and viable bacteria are rare.

\section{Introduction}

Characterizing the very first intestinal bacteria is essential for a better understanding of the codevelopment of the newborn and its intestinal microbiome. Host-microbiome interactions enable early-life education and maturation of the immune system during a window of opportunity. The use of prenatal and intrapartum antibiotics may disturb this process (Dierikx et al., 2020). However, the timing of the microbial colonization of the mammalian gut is still unclear (Perez-Munoz et al., 2017; Korpela and de Vos, 2018; Liu et al., 2019; Guzman et al., 2020). Vertical as well as environmental transmission of bacteria occur during and after birth and seed the neonatal gastrointestinal (GI) tract (Funkhouser and Bordenstein, 2013; Korpela and de Vos, 2018). Transmission of an orally inoculated Enterococcus faecium strain in pregnant mice to the meconium of their fetuses has been described (Jimenez et al., 2008).

Recent studies in humans, mice and cattle have reported the identification of microbial DNA or culturable bacteria in the mammalian fetal environment (Aagaard et al., 2014; Severovic et al., 2019; Al Alam et al., 2020; Rackaityte et al., 2020; Guzman et al., 2020). Nevertheless, others interpret such observations as intrauterine infections or contamination (Lauder et al., 2016; Eisenhofer et al., 2018; de Goffau et al., 2019; Hockney et al., 2020; Olomu et al., 2020; Theis et al., 2020a, 2020b). The newborn's GI tract is a low microbial biomass environment, implying multiple challenges for performing reliable microbiome analyses (Glassing et al., 2016; Eisenhofer et al., 2018; Stinson et al., 2018). Besides the technical limitations inherent to DNA sequencing, there are practical and ethical concerns while collecting samples in 
humans. First-pass meconium has been used as a proxy to assess the fetal intestinal microbiome, although samples are often collected hours after birth and even breastfeeding, suggesting potential postnatal effects on the microbial composition of the meconium (Collado et al., 2016; Liu et al., 2019; Stinson et al., 2019). Mammalian animal models can overcome several of these ethical and practical difficulties, providing insights in the fetal gastrointestinal microbiome.

In the current study, the microbial composition of the full-term fetal gut and the corresponding amniotic fluid was assessed in Belgian Blue cow-calf couples. In this double-muscled beef breed, caesarean sections (C-sections) are performed on a routine basis during the very early stages of parturition, while fetal membranes are still intact, rendering this breed a highly suitable model for full-term gestation microbiome studies.

Besides the potential role of the cow model for research, there is also a significant interest in the composition and the development of the calf's intestinal microbiome. Gut health and growth performance during the first weeks of life are main drivers for cost effective livestock rearing (Thornton Philip, 2010; Lorenz et al., 2011). Few studies have been conducted on the intestinal microbiome in vaginally born neonatal calves. These have reported low numbers of bacteria, mostly belonging to the phyla Proteobacteria, Firmicutes, Actinobacteria and

66 Bacteroidetes (Alipour et al., 2018; Klein-Jöbstl et al., 2019; Guzman et al., 2020). Shared microbiota were found between calf meconium and the maternal vaginal vestibulum (Alipour et al., 2018; Yeoman et al., 2018; Klein-Jöbstl et al., 2019). To the best of our knowledge, no microbiome studies have yet been performed on calves born by $\mathrm{C}$-section, and no studies are available to assess the association between the microbiome of the calf's meconium and the microbiome of the corresponding amniotic fluid. 
72 Our aims were to assess the bacterial load and characterize the bacterial composition of the

73 amniotic fluid and meconium of full-term neonatal calves. To this end, samples were collected

74 during elective C-section, and analyzed by qPCR, bacterial culture under carefully controlled and validated conditions, and 16S rRNA gene amplicon sequencing. To minimize reagent and sequences.

\section{Materials and methods}

\section{Study design} were housed for an elective C-section.

environmental bacterial DNA contaminants, we included multiple technical controls and stringently filtered the $16 \mathrm{~S}$ rRNA gene sequencing data to exclude putative contaminant

All experimental procedures were approved by the institutional ethics and animal welfare committee of the Faculty of Veterinary Medicine (EC2018/002 - Ghent University, Belgium).

The cows' owners were informed about the study and gave their written consent.

The sampling of this study was performed at the teaching hospital of the Department of Reproduction, Obstetrics, and Herd Health of the Faculty of Veterinary Medicine in Ghent (Belgium), where pregnant Belgian Blue beef cows of different herds (parity between 1 and 5)

In total 25 Belgian Blue cows and their calves ( 7 males and 18 females) were sampled from November 2017 until March 2019. The cows were housed in tie-stalls at the facility for $9.5 \mathrm{~d}$ \pm 5.8 (mean \pm standard deviation) prior to $\mathrm{C}$-section and had ad libitum access to hay and water. During this period, rectal temperature was measured twice daily, and calving indicators such as udder distension, teat filling, pelvic ligament relaxation, vaginal discharge, vulvar edema, and behavioral changes were monitored every two hours by graduate veterinary students. 
94 Prior to elective C-section, in cows that exhibited a drop in temperature, cervical dilation was

assessed by manual palpation. The vulvar region was cleaned with iodine soap and water. A gloved hand was inserted vaginally and the opening of the portio vaginalis cervicis was estimated. For the present study, elective C-section was performed when the cow had a minimal cervical dilation of eight centimeters, with no rupture of the fetal membranes prior to surgery. All cows were healthy according to their vital parameters (heart rate, temperature, respiratory rate) and there was no clinical evidence of intrauterine infection or contamination.

\section{Sampling}

Prior to surgery, the cows were restrained in a standing position in a surgery crush specifically designed for cattle. C-section procedure was done as described by Kolkman et al. (2007). Briefly, the surgical area (left flank) was washed and disinfected, an abdominal incision was made, and part of the uterus was exteriorized for uterotomy. The allantoic sac was opened up to expose the intact amniotic sac. Amniotic fluid was aspirated through the amniotic membrane, using a sterile $16 \mathrm{G}$ needle (Agani, Terumo Europe, Hamburg, Germany) and sterile $20 \mathrm{ml}$ syringe (B. Braun, Melsungen, Germany). Within one hour after sampling, the retrieved volume was aliquoted, under a laminar-flow hood, into 2 sterile $15 \mathrm{ml}$ tubes (188271, Cellstar, Greiner bio-one, Frickenhausen, Germany). In the first tube, $6 \mathrm{ml}$ of amniotic fluid was dissolved in $3 \mathrm{ml}$ of glycerol ( $\geq 99 \%$, G2025, Sigma-Aldrich (Merck), Overijse, Belgium) and subsequently stored at $-80{ }^{\circ} \mathrm{C}$ to be used in culture experiments. In the second tube, $12 \mathrm{ml}$ of amniotic fluid was stored at $-80^{\circ} \mathrm{C}$ to be used for bacterial DNA extraction.

Meconium samples were acquired directly from the calves' rectum, immediately after birth (no more than 30 minutes). Until the moment of sampling, calves laid on a clean concrete floor, with access to neither the dam, nor colostrum. The perineum of the calf was dried with a clean paper towel and disinfected with $70 \%$ ethanol. A sterile double-guarded equine uterine culture 

a sterile $2 \mathrm{ml}$ cryovial containing $1 \mathrm{ml}$ of a $30 \%$ glycerol solution, prepared by diluting glycerol ( $\geq 99 \%$, G2025, Sigma-Aldrich (Merck), Overijse, Belgium), in ultra-pure, nuclease-free water (W4502, Sigma-Aldrich (Merck), Overijse, Belgium) to a final $30 \%$ concentration.

Negative field controls were processed in the surgery room, using the same sampling procedures and disposables. In total, 16 empty, sterile double-guarded equine uterine culture swabs (10 Har-vet swabs and 6 Minitube swabs) were included for the meconium sampling, 5 of the Har-vet swabs stored in $1 \mathrm{ml}$ of a $30 \%$ glycerol stock solution, the others with no additives. Additionally, 12 negative controls were included for the amniotic fluid sampling, aspirating ultra-pure, nuclease-free water (W4502, Sigma-Aldrich (Merck), Overijse, Belgium) instead of amniotic fluid. For 8 of them, the ultra-pure, nuclease-free water was stored with no glycerol.

133 All samples were shipped on dry ice to the laboratory of the Department of Veterinary

134 Biosciences of the Faculty of Veterinary Medicine in Helsinki (Finland) for further processing.

135 Culture

\section{Validation of culture media}

137 One of our aims was to define a single bacterial culture medium, capable of sustaining a majority of the calf intestinal core microbiota. Consequently, this single medium could be used

139 for culturing the meconium and amniotic fluid samples, avoiding further splitting or dilution of the low biomass samples. We first tested GAM "Nissui" (Gifu Anaerobic Medium Agar, 
141 Code 05420, HyServe, Germany), YCFA (Yeast extract, Casitone and Fatty Acid), LB

142 (Lysogeny Broth, BD) and BB (Trypticase Soy Agar supplemented with Bovine Blood 211043,

143 Tammer BioLab Oy, Tampere, Finland) media for the ability to sustain calf intestinal core

144 microbiota, by plating each type of medium anaerobically with feces of a healthy 7 day old calf

145 (Lopez-Siles et al., 2012; Alipour et al., 2018). After 7 days of growth, mixed cultures were 146 extracted from the plates, 16S rRNA gene amplicon sequenced, and compared to the core

147 microbiota previously observed in fecal samples of young calves (Alipour et al., 2018).

\section{Sample culturing}

149 Bacterial culture on GAM-agar plates was performed for 24 meconium samples (5 corresponding negative controls) and 24 amnion samples (4 negative controls). The frozen samples were first transferred to an anaerobic workstation (Ruskinn Concept Plus), mixed thoroughly and plated, using an aseptic technique, at $+37^{\circ} \mathrm{C}$. After this, the samples were transferred to a laminar flow cabinet and plated in aerobic conditions at $+37^{\circ} \mathrm{C}$. The culture plates were checked for growth daily for 14 days. All visible bacterial colonies were subcultured until pure isolates were obtained. Fresh cultures were then identified using MALDI-TOF (Bruker Microflex LT) at the Central laboratory of the Faculty of Veterinary Medicine (Helsinki, Finland). Mass spectra were analyzed in a mass/charge range from 2000 to 20000 Da with MBT

160 Compass v4.1 on flexControl v3.4 (Bruker Daltonik GmbH) using BDAL-7311 as the reference library. The Bruker Bacterial Test Standard (RUO) (Bruker Daltonik GmbH) was used for instrument calibration. If the identification confidence score was $<2.00$, further identification was done with 16S rRNA gene amplicon Sanger sequencing at the Institute of

164 Biotechnology (University of Helsinki, Finland). 
DNA from the meconium samples $(\mathrm{N}=25)$ and corresponding negative controls $(\mathrm{N}=11)$ were according to the manufacturer's instructions, with minor modifications to the protocol as described previously (Alipour et al., 2018; Husso et al., 2020). ZymoBIOMICS ${ }^{\text {TM }}$ Microbial Community Standard and an in-house fecal standard were processed with the meconium samples and no-template-controls were included in every batch.

172 For the amniotic fluid samples $(\mathrm{N}=23)$ and the corresponding negative controls $(\mathrm{N}=8), 2 \mathrm{ml}$ 173 of each sample was first centrifuged in a microcentrifuge at $16100 \times \mathrm{g} 10 \mathrm{~min}$ in $+4{ }^{\circ} \mathrm{C}$. Most 174 of the supernatant was removed and $750 \mu 1$ of ZymoBIOMICS Lysis Solution and $19 \mu 1$ of 175 proteinase K (D3001-2-20/D3001-2-5, Zymo Research) were added to the remaining $200 \mu 1$ of the samples and incubated for $30 \mathrm{~min}$ at $+55^{\circ} \mathrm{C}$. After these steps, the same protocol as applied for the meconium samples was followed.

All manipulations of the tubes during the process were performed in a laminar flow cabinet, and the workplace, instruments and pipettes were cleaned routinely with $10 \%$ bleach. Certified DNA, RNase, DNase and PCR inhibitor free tubes (STARLAB International, Germany) and Nuclease-free Water (Ambion, Thermo Fisher Scientific, USA) were used for DNA extraction and downstream analyses. All extracted DNA was stored at $-80^{\circ} \mathrm{C}$.

\section{Quantitative PCR}

184 The bacterial 16S rRNA gene copy numbers in the meconium, amnion and negative control samples were determined using quantitative PCR. The analyses were performed as described previously (Alipour et al., 2018; Husso et al., 2020), with the exception that the PCR master 
mix was first treated using a dsDNAse based decontamination kit (Enzo Life Sciences,

188 Farmingdale, New York). The amplification was performed using the Bio-Rad CFX96 instrument (Bio-Rad, Hercules, California) and universal eubacteria probe and primers

190 (Nadkarni et al., 2002). A standard series and negative controls were included in every run.

191 The data were analyzed using the Bio-Rad CFX Maestro software. The results were normalized to the averages of absolute 16S rRNA gene copy numbers in the negative controls included in each run.

\section{Library preparation and 16S rRNA gene amplicon sequencing}

The V3-V4 region of the 16S rRNA gene amplicons was sequenced using the Illumina MiSeq platform in the DNA core facility of the University of Helsinki, as described previously (Alipour et al., 2018; Husso et al., 2020). In total, 23 amniotic fluid samples and 23 meconium samples of the same cow-calf couple were sequenced. adult cow fecal standard were pre-amplified with 12 cycles and all other sample types, including negative controls, with 21 cycles.

202 The observed composition and abundances for the commercial standard matched the expected 203 composition provided by the manufacturer (data not shown).

\section{Bioinformatics}

205 The detailed bioinformatics pipeline is described in the Supplementary materials. Briefly, the read quality was first inspected with FastQC and MultiQC (Andrews, 2011; Ewels et al., 2016).

207 Leftover primes and spacers were then trimmed with Cutadapt v1.10 (Martin, 2011). 208 A mapping file was created for QIIME2 and validated with Keemei (Rideout et al., 2016). The 
FASTQ-files were imported to QIIME2 v2019.4, where the DADA2 plugin was used to denoise and quality filter the reads, call ASVs and generate a feature table (Callahan et al.,

211 2016; Bolyen et al., 2019). A naïve Bayes classifier was trained in QIIME2 against SILVA 212 v132 $99 \%$ database, extracted to only include the V3-V4 region and used to assign taxonomy 213 to ASVs (Quast et al., 2013; Bokulich et al., 2018). Sequences derived from chloroplasts or 214 mitochondria were removed and singletons were filtered out, leaving only bacteria with at least 215 phylum-level identification.

216 The processed data was in silico filtered to remove ASVs which represented probable

217 contaminants, as described previously (Husso et al., 2020). Briefly, an ASV was removed if its 218 prevalence in actual samples was $\leq 2 \times$ its prevalence in instrument controls (DNA extracted 219 from empty sampling instruments only handled aseptically in a laminar flood cabinet) or its prevalence in field controls (empty sampling instruments exposed to the surgery room environment), and if its mean relative abundance in actual samples was $\leq 10 \times$ its mean abundance in instrument controls or its mean abundance in field controls. The filtering was performed separately for meconium and amnion samples. If less than 500 reads remained after

224 the decontamination, as was the case for six meconium samples, the sample was removed from 225 further analyses.

\section{Statistics}

227 The 16S rRNA gene qPCR results from samples and negative controls were compared using two-tailed Mann-Whitney U test in IBM SPSS Statistics 25.

229 Shannon diversity indices were calculated for genus-level data with the R package phyloseq 230 (McMurdie and Holmes, 2013). The Kruskal-Wallis rank sum test was used to compare the bacterial community structure of both sample types in RStudio (R Studio Team, 2020). 
The PCoA figures were plotted using ASV and genus-level data and Bray-Curtis distances with

calculated using the R package vegan, using 9999 permutations (Oksanen et al., 2019).

236 DESeq2 was used to explore differentially abundant ASVS by calculating differential 237 expression between sample groups (Love et al., 2014).

238 The LEfSe (Linear discriminant analysis Effect Size) web application was used to identify the 239 taxons most likely explaining the differences between the sample types (Segata et al., 2011).

240 An ecologically organized heatmap of the top 40 most abundant ASVs was created with the R

241 package phyloseq (Rajaram and Oono, 2010; McMurdie and Holmes, 2013).

242 Spearman correlations and their Bonferroni corrected p-values were calculated using R243 package Hmisc 4.3.0 (Harrel, 2019).

244 Differences were considered significant at $\mathrm{P}<0.05$.

\section{Results}

246 Quantification of bacterial 16S rRNA in amniotic fluid and meconium

247 samples by qPCR

248 The 16S rRNA gene copy numbers in amniotic fluid and meconium samples were assessed by 249 qPCR (Fig. 1). To minimize variation between qPCR runs, the copy numbers were normalized 250 to the averages of the negative controls in the same run as explained in Materials and Methods. 
In the amniotic fluid samples, there was no significant difference $(P=0.104)$ between the

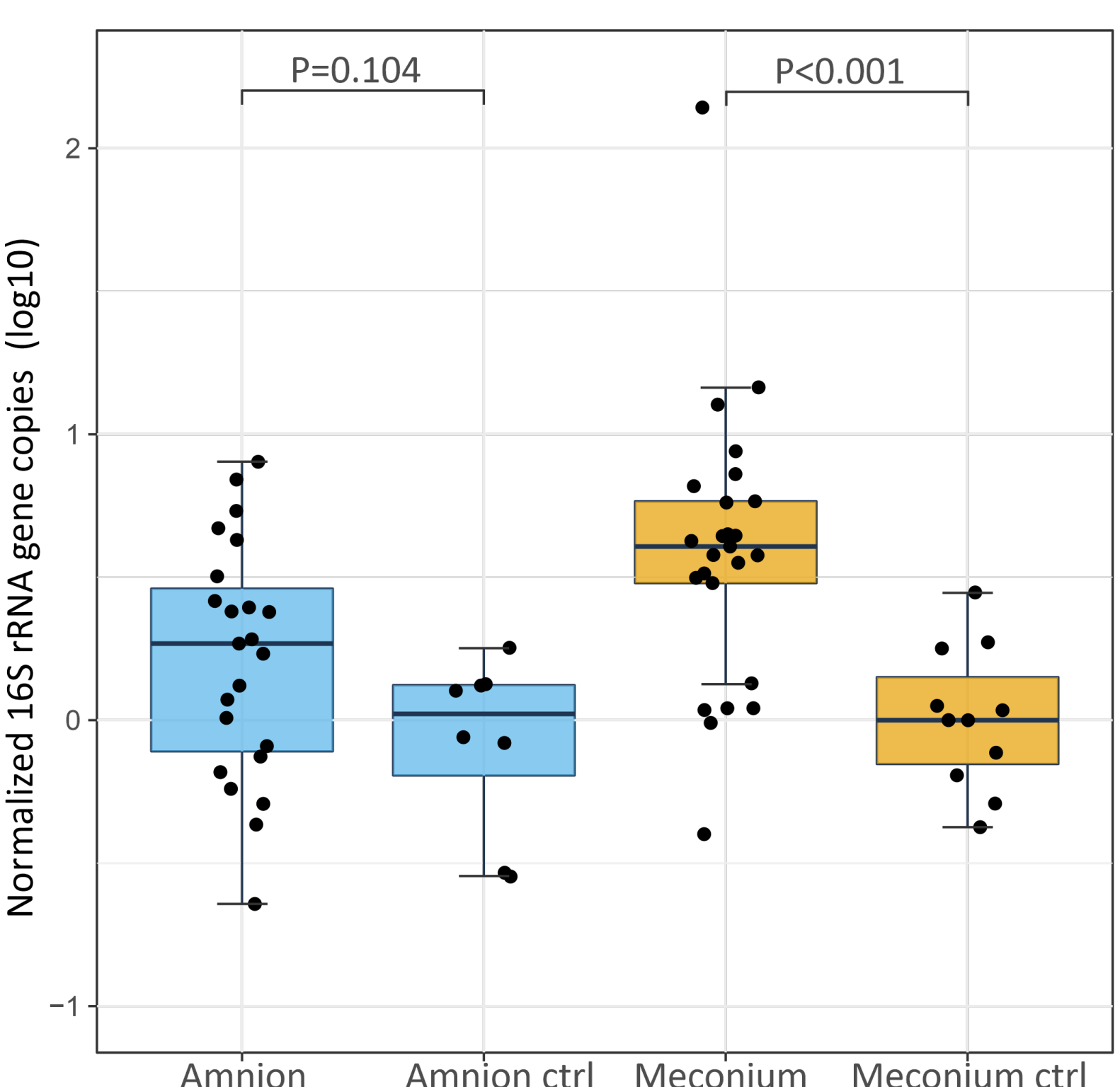
negative controls $n=8)$ and meconium samples $(n=25$, negative controls $n=11)$. Samples were collected during elective C-section in Belgian Blue cow-calf pairs. The copy number of each sample was normalized to the average copy number of the corresponding negative controls and $\log 10$ 
transformed. The midline of the box is the median, with the upper and lower limits of the box being the third and first quartile. The whiskers extend up to 1.5 times the interquartile range from the box to the 265 furthest data point within that distance.

\section{Decontamination of the 16S rRNA gene amplicon sequencing data}

267 We performed a stringent filtering of the 16S rRNA gene sequencing data to remove the amplicon sequence variants (ASVs) potentially stemming from reagent contaminants and environmental bacterial DNA (Table 1).

The decontamination was based on the prevalence and relative abundance of each ASV in the samples and negative controls (see Materials and Methods).

Table 1. Mean read counts (standard deviation) detected in meconium and amniotic fluid samples and their negative controls. Only sequences with at least phylum-level identification were retained for further analyses. If less than 500 reads remained after in silico decontamination, as in six meconium samples, the sample was removed from further analyses.

\begin{tabular}{l|l|l|l|l|l|l}
$\begin{array}{l}\text { Sample } \\
\text { type }\end{array}$ & \multicolumn{2}{l}{ Raw } & \multicolumn{2}{l}{ Processed } & \multicolumn{2}{l}{ Decontaminated } \\
\hline & $\mathrm{N}$ & Reads & $\mathrm{N}$ & Reads & $\mathrm{N}$ & Reads \\
\hline Meconium & 23 & $\begin{array}{l}92266 \\
(36235)\end{array}$ & 23 & $\begin{array}{l}57082 \\
(25543)\end{array}$ & 17 & $\begin{array}{l}4189 \\
(8931)\end{array}$ \\
\hline $\begin{array}{l}\text { Meconium } \\
\text { control }\end{array}$ & 11 & $\begin{array}{l}126800 \\
(21164)\end{array}$ & 11 & $\begin{array}{l}84023 \\
(14585)\end{array}$ & & - \\
\hline Amnion & 23 & $\begin{array}{l}127797 \\
(22376)\end{array}$ & 23 & $\begin{array}{l}83279 \\
(15120)\end{array}$ & 23 & $\begin{array}{l}3338 \\
(2922)\end{array}$ \\
\hline $\begin{array}{l}\text { Amnion } \\
\text { control }\end{array}$ & 6 & $\begin{array}{l}136488 \\
(12663)\end{array}$ & 6 & $\begin{array}{l}90802 \\
(8998)\end{array}$ & & - \\
\end{tabular}

\section{Microbial composition of amniotic fluid and meconium samples}

278 The microbiota of both sample types consisted primarily of Proteobacteria, Firmicutes, 279 Bacteroidetes and Actinobacteria phyla, with individual variation (Fig. 2). In both sample types, the inter-individual variation increased at lower taxonomic levels (Supplementary Table 
bioRxiv preprint doi: https://doi.org/10.1101/2020.09.28.309476; this version posted November 4, 2020. The copyright holder for this preprint (which was not certified by peer review) is the author/funder, who has granted bioRxiv a license to display the preprint in perpetuity. It is made available under aCC-BY 4.0 International license.

1). In the amniotic fluid samples, Staphylococcus, Streptococcus, Delftia, Sphingomonas and

Enterococcus were the most abundant genera, and Delftia, Streptococcus, Staphylococcus,

Sphingomonas and Acinetobacter were the most prevalent genera (Supplementary Table 1). composition provided by the manufacturer.

289
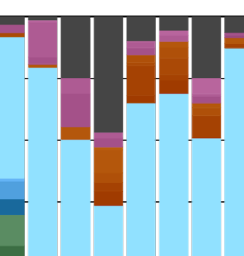
Non

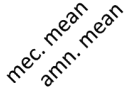
Proteobact.

Firmicutes Bacteroid.
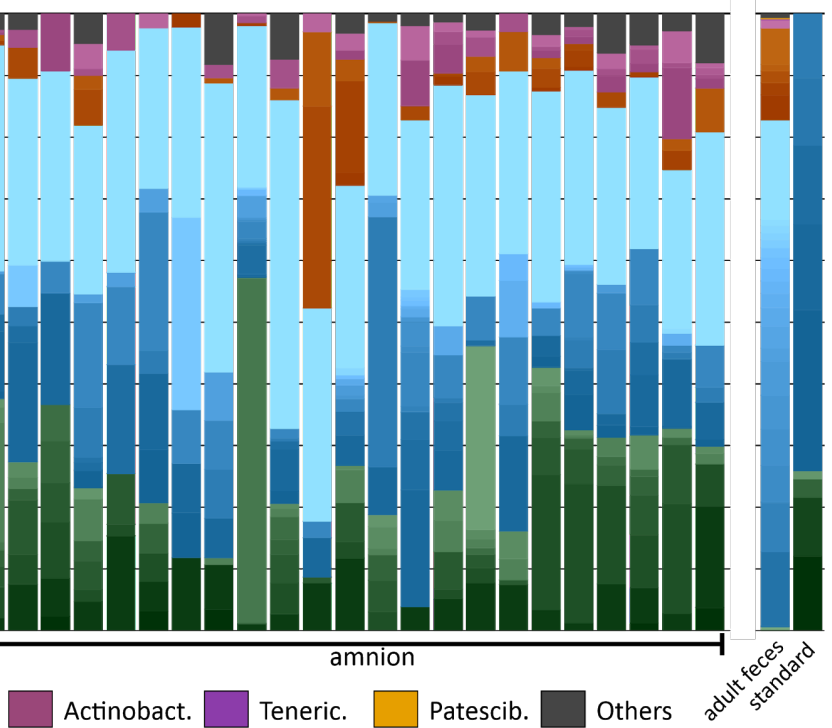

Figure 2. Microbiota composition in bovine meconium and amniotic fluid samples collected during

elective $C$-section, adult feces, and a commercial community composition standard. The main colors indicate the bacterial phyla. Within phyla, the shades indicate bacterial genera. The lightest shade of each phylum shows the combined abundance of the least abundant genera (with a maximum of $<0.5 \%$ of total).

A heatmap analysis of the 40 most abundant ASVs shows the difference between the sample types in more detail (Fig. 3). 
bioRxiv preprint doi: https://doi.org/10.1101/2020.09.28.309476; this version posted November 4, 2020. The copyright holder for this

preprint (which was not certified by peer review) is the author/funder, who has granted bioRxiv a license to display the preprint in perpetuity. It is made available under aCC-BY 4.0 International license.

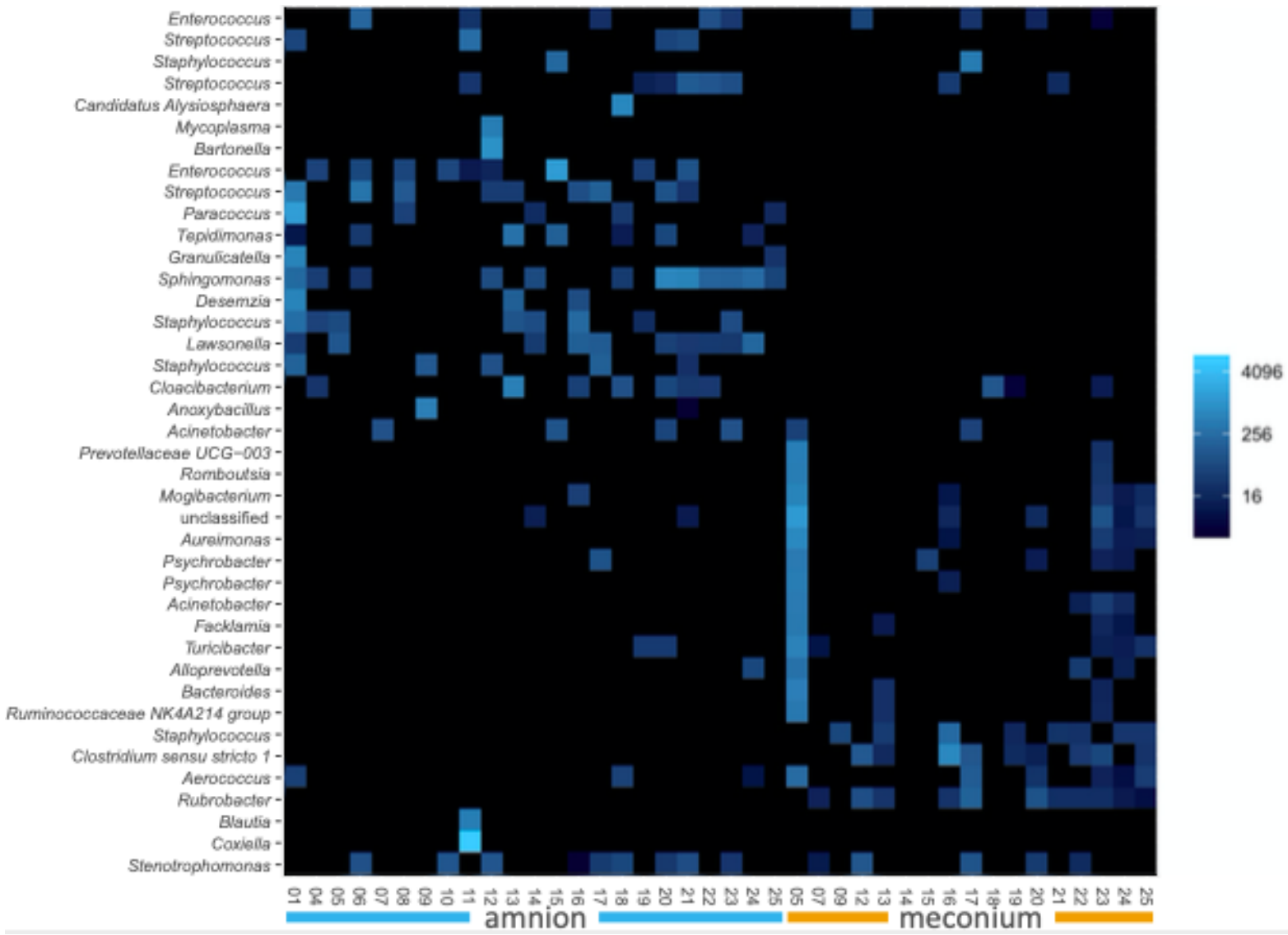

301 Figure 3. Ecologically organized heatmap (NMDS, Bray) of the 40 most abundant ASVs in bovine

302 meconium and amniotic fluid samples collected during elective $C$-section, sorted by sample type. The 303 taxon names are presented as genus level identifications. Color scale indicates relative abundance and 304 is a log transformation with base 4.

305 The alpha diversity (Shannon index) at the genus level was not significantly different between 306 the meconium and amniotic fluid samples (Fig. 4, $\mathrm{P}=0.889$ ). 


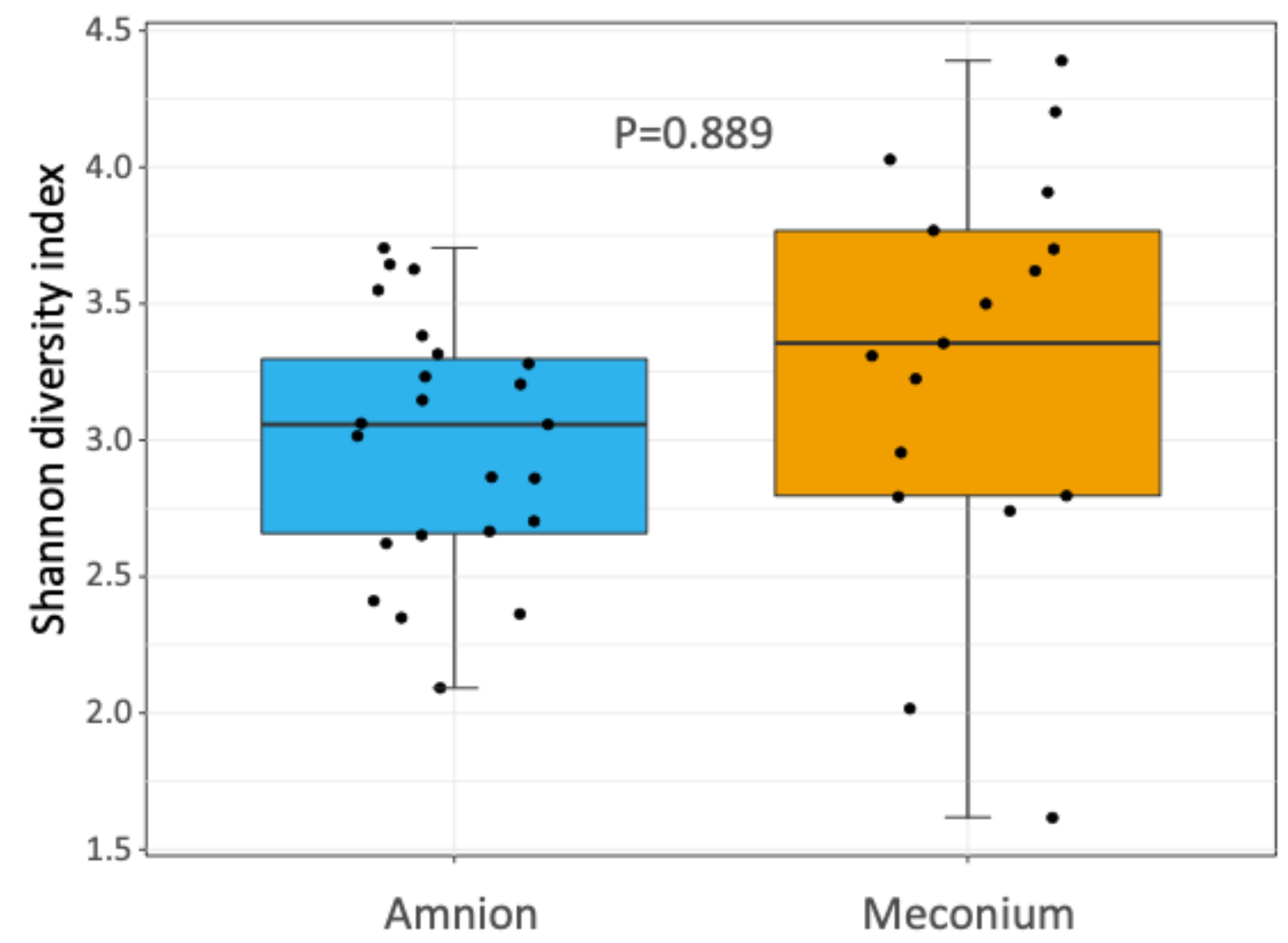

309 Figure 4. Shannon diversity index showing the bacterial community structure (at genus level) of pairs. Boxplot as in Fig 1. 


\section{Comparison of amniotic fluid and meconium microbiota composition}

317 The microbiota composition was significantly different for amniotic fluid and meconium 318 samples at the ASV (PERMANOVA, $\mathrm{P}<0.001, \mathrm{R} 2=0.049$, Fig. $5 \mathrm{~A}$ ), and genus level 319 (PERMANOVA, P < 0.001, R2 =0.062, Fig. 5 B).
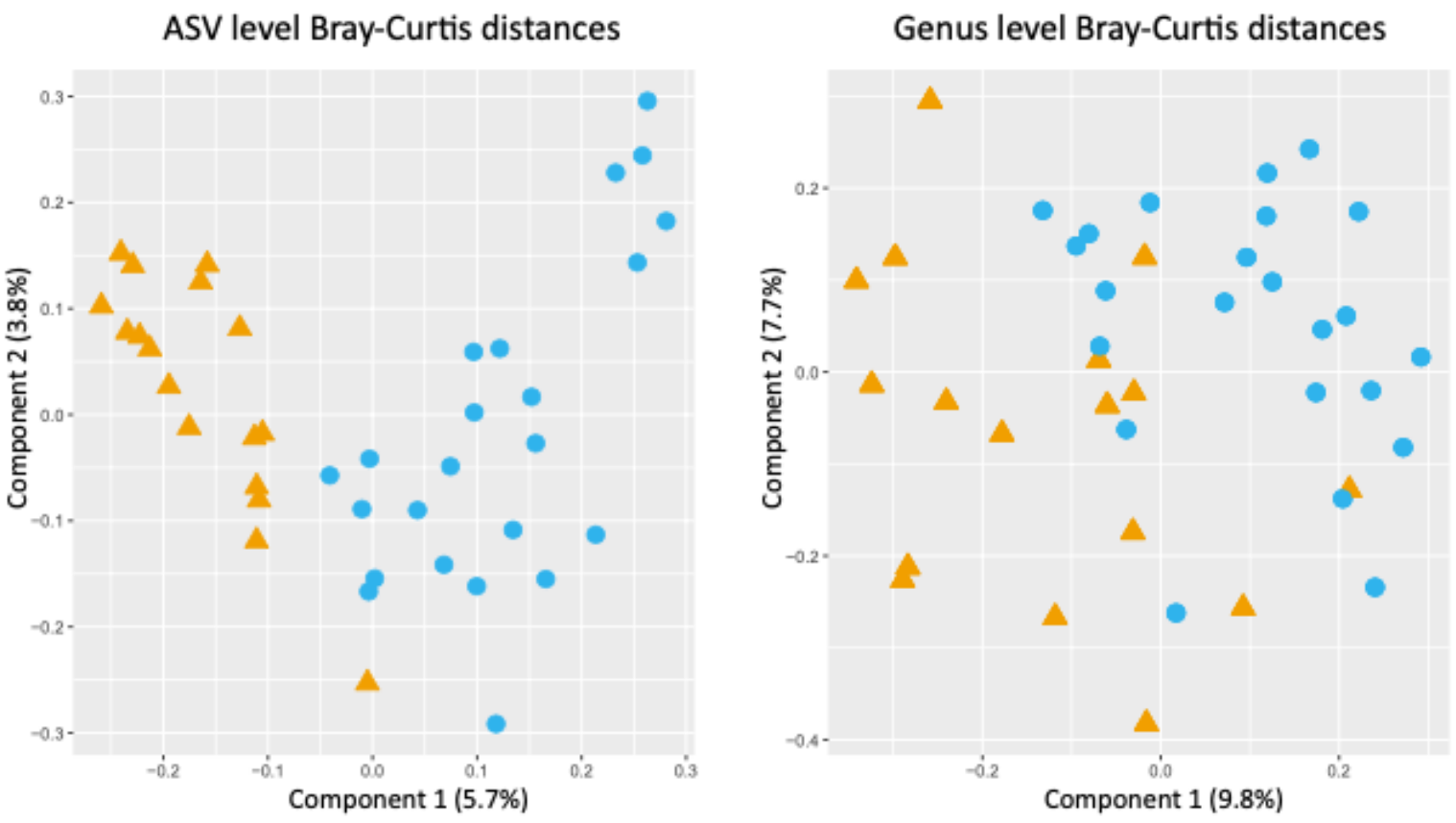

Sample types: amnion $\Delta$ meconium

Figure 5. Comparison of the bacterial community structure of amniotic fluid and meconium. 5 A: PCoA on Bray-Curtis distances based on ASV level data. Figure 5 B: PCoA on Bray-Curtis distances based on genus level data. Colors and shapes indicate the sample types.

325 According to the DESeq2 analysis, three ASVs were more abundant in meconium than in amniotic fluid: one Staphylococcus ASV ( $\log 2$ Fold Change $\left.=25.084, \mathrm{P}_{\mathrm{adj}}<0.001\right)$, one 
In amniotic fluid, one Sphingomonas ASV (log2 Fold Change $\left.=-8.420, \mathrm{P}_{\mathrm{adj}}<0.001\right)$ and one meconium.

By LefSe (Linear discriminant analysis effect size) analysis including all taxonomic levels, meconium samples had a greater relative abundance of Clostridiales and Rubrobacter (LDA score $(\log 10)>4.0)$ than the amniotic fluid, but a lower relative abundance of Bacillales and

We calculated Spearman rank correlations for the microbiota between meconium and amniotic

Average correlations $(\rho)$ at $\operatorname{ASV}\left(\mathrm{N}=17, \rho_{\mathrm{avg}}=-0.0201, \mathrm{P}_{\mathrm{avg}}<0.05\right)$ and genus level $(\mathrm{N}=17$,

$\left.341 \rho_{\text {avg }}=0.1685, \mathrm{P}_{\text {avg }}<0.05\right)$ were very weak and biologically not significant. All p-values were

342 Bonferroni corrected for multiple testing.

\section{Bacterial cultures}

344 Based on our testing of multiple media, the GAM (Gifu Anaerobic Medium) agar was selected 345 to assess if there were live bacteria in the samples stored in glycerol (see Materials and

346 Methods), since it was able to sustain the largest number of bacterial core genera found in calf

347 feces, including some additional unique genera not found on any other plate type (Alipour et al., 2018).

349 All amniotic samples and their negative controls were culture negative, both in anaerobic and aerobic conditions. Five out of 24 meconium samples and 1 out of 5 negative controls were 
culture positive on GAM agar. The results for each sample are presented in detail in Table 2.

352 Both gram negative and gram-positive strains were isolated from the samples.

Table 2. Culture positive meconium samples and identified bacteria. If the identification confidence score was $<2.00$ for MALDI-TOF, further identification was done with 16S rRNA gene amplicon Sanger sequencing to achieve genus level identification for all isolates.

\begin{tabular}{|c|c|c|}
\hline Sample & Aerobic strains & Anaerobic strains \\
\hline Negative control 1 & $\begin{array}{c}\text { Staphylococcus sp. }(\mathrm{Gram}+) \\
\text { Kocuria } \mathrm{sp} .(\mathrm{Gram}+)\end{array}$ & Rothia sp. (Gram+) \\
\hline Meconium 5 & Roseomonas sp. (Gram-) & - \\
\hline Meconium 10 & Acinetobacter sp. (Gram-) & $\begin{array}{l}\text { Streptococcus sp. (Gram+), } \\
\text { Fusobacterium sp. (Gram-) }\end{array}$ \\
\hline Meconium 12 & - & Cutibacterium sp. (Gram+) \\
\hline Meconium 16 & Kocuria sp. (Gram+) & - \\
\hline Meconium 21 & Achromobacter sp. (Gram-) & - \\
\hline
\end{tabular}

357 The Sanger sequence from the Roseomonas sp. isolate acquired from a meconium sample was a $100 \%$ identical match to the $16 \mathrm{~S}$ rRNA gene amplicon sequencing data from the same sample. In addition, the Sanger sequences of Staphylococcus sp., Kocuria sp., Roseomonas sp., and Acinetobacter sp. isolates matched the 16S rRNA gene amplicon sequencing data when compared to all meconium samples. 

samples.

\section{Discussion}

366 Vertical transmission of microbes during, and perhaps already before birth is a universal 367 phenomenon across the whole animal kingdom and plays an important role in shaping host 368 immunity and fitness (Funkhouser and Bordenstein, 2013; Vandenplas et al., 2020). Despite

369 the advances in our understanding of the assembly and lifelong effects of the perinatal microbiota, the in utero exposure of the fetus to maternal bacteria and bacterial components remains poorly understood (Perez-Munoz et al., 2017; Korpela and de Vos, 2018; Liu et al., 2019).

In the present study, we sampled amniotic fluid during, and the corresponding calves

374 immediately after they were born by elective $\mathrm{C}$-section. The elective $\mathrm{C}$-section is a routine

375 procedure in Belgian Blue beef cattle, since the pelvis of the dam does not allow vaginal birth of the relatively large double-muscled calf (Kolkman et al., 2007). We performed the surgery

377 during the very early stages of parturition, when both fetal membranes (amniotic and allantochorionic membrane) were still intact, allowing us to sample the full-term fetus and the intrauterine environment without exposure to the vaginal microbes and minimal exposure to 380 the environmental microbiota. Meconium samples were collected rectally. The relatively large size of the calves permits the insertion of a double-guarded sampling device into the rectum, minimizing the risk of contamination. 
meconium is often collected several hours after birth, microbes from the environment and the transmission of bacteria to the neonatal gastrointestinal tract (Dornelles et al., 2020). In the current study, amniotic fluid samples were collected in vivo, while the amniotic membrane was still intact, as has been done in humans during pre-labor C-section (Collado et al., 2016).

Bacterial culture is traditionally used to determine the viable population in a bacterial different selective media and multiple specific growing conditions (Lau et al., 2016; Lagier et al., 2018). In the current study, one of our aims was to define a single bacterial culture medium, capable of sustaining the majority of the calf intestinal core microbiota. We first tested four different culture media: GAM "Nissui" (Gifu Anaerobic Medium Agar), mostly used for anaerobic bacteria, YCFA (Yeast extract, Casitone and Fatty Acid), containing volatile fatty acids typically supporting the growth of several gut bacteria, LB (Lysogeny Broth) as a general media, and BB (Trypticase Soy Agar supplemented with Bovine Blood) supporting fastidious bacteria by blood enrichment. The GAM medium was found optimal for both aerobic and anaerobic culturing.

Bacteria were successfully cultured from 5 out of 24 meconium samples, representing both gram-negative and gram-positive bacterial genera, known to live in mammalian hosts. Roseomonas and Achromobacter species are known to act as opportunistic pathogens, but have 406 also been isolated from a wide variety of environmental sources (Reverdy et al., 1984; Rihs et 407 al., 1993). Various Acinetobacter and Streptococcus species have physiological functions in their mammalian hosts, but the genera also include pathogenic and/or environmental species 
409 (Krzyściak et al., 2013; Touchon et al., 2014). Fusobacteria species are typically closely related

410 to mucous membranes, especially the oral cavities, while Cutibacterium and Kocuria species

411 have most often been isolated from the skin (Hofstad, 1992; Savini et al., 2010; Scholz and

412 Kilian, 2016). One of our negative controls yielded Kocuria colonies. This was the only taxon

413 shared between the actual meconium samples and the negative controls, suggesting low-level contamination from the sampling environment.

415 Although we detected cultivable bacteria in some of the meconium samples, most of the samples were culture negative. This indicates that the presence of live bacteria in the fetal

417 intestine is rare, in contrast to the prevalent bacterial DNA. Individual differences in amounts

418 of viable bacteria retrieved from meconium were also described in a recent dog study.

419 Interestingly, puppies born without detectable meconium microbiota were shown to have a slower growth rate than those in which meconium microbiota were detected (Pipan et al., 2020). Both the mucosal surface of the intestine as well as the amniotic fluid contain bacteriostatic substances, such as lactoferrin and salivary scavenger and agglutinin (SALSA), which are able to suppress bacterial growth and viability (Reichhardt and Meri, 2016;

424 Lisowska-Myjak et al., 2019). In human meconium, SALSA amounts to $10 \%$ of all proteins, highlighting its potential role in antimicrobial defense (Reichhardt et al., 2014). Lactoferrin, SALSA and other similar substances may explain the small number of viable bacteria observed in our study, in contrast to the significant amount of detected bacterial DNA. contamination is a major challenge in the analysis of low bacterial biomass samples, we

430 processed several types of negative controls alongside our biological samples, treated qPCR mastermix with dsDNAse, and applied rigorous in silico filtering of potential contaminants 432 (Glassing et al., 2016; Eisenhofer et al., 2018). Many similar studies are published without or 
with a very limited number of contamination controls, which makes our approach more reliable, especially for assessing low bacterial biomass environments.

435 We observed a small but significant amount of bacterial DNA in the meconium samples by 16S qPCR, suggesting a prenatal transmission of bacterial DNA to the fetal intestine. This is consistent with previous studies in cattle, in which calves born at term per vias naturales, were sampled (Mayer et al., 2012; Alipour et al., 2018; Klein-Jöbstl et al., 2019). A profile dominated by the phyla Proteobacteria, Firmicutes, Bacteroidetes and Actinobacteria was identified in the meconium, in agreement with previous studies using 16S rRNA gene amplicon sequencing (Alipour et al., 2018; Yeoman et al., 2018; Klein-Jöbstl et al., 2019; Husso et al., 2020; Guzman et al., 2020). Additionally, some of the most prevalent genera observed in meconium in our study, such as Sphingomonas, Acinetobacter, Pseudomonas, Ruminococcus and Stenotrophomonas, have also been previously described in neonatal calves (Klein-Jöbstl et al., 2019).

In contrast to the meconium, there was no significant difference between the 16S rRNA gene copy numbers of amniotic fluid samples and the negative controls, and all were culture negative. A recent caesarean section study in sheep stated amniotic fluid sterility during the 449 third trimester of pregnancy (Malmuthuge and Griebel, 2018). In other studies however, a low biomass microbiome in the pregnant bovine uterus was described (Moore et al., 2017; Guzman et al., 2020).

452 Using 16S rRNA gene amplicon sequencing, we found a bacterial signature in the amniotic fluid, dominated by the phyla Proteobacteria, Firmicutes, Bacteroidetes and Actinobacteria, in accordance with previous studies (Karstrup et al., 2017; Moore et al., 2017; Guzman et al., 2020). Deeper taxonomic identification, down to the family or genus level, revealed no further agreements with these studies. 
From a comparative point of view between mammalian species, the amniotic fluid sampled by

458 Collado et al. (2016) in women during pre-labor C-section was found to contain a distinct, low

Despite this and other recent studies, the presence of microbial DNA and culturable bacteria in the human placenta and fetal environment is still under debate (de Goffau et al., 2019).

The microbial DNA signatures in meconium and amniotic fluid were similar at the phylum

463 level. However, they presented a significantly different microbial composition at genus and 464 ASV level: genera Staphylococcus, Rubrobacter and Clostridium were relatively more abundant in meconium samples than in amniotic fluid samples according to DESeq2 analysis.

466 Complementing this with the LefSe analysis using all taxonomic levels, also found Clostridiales and Rubrobacter taxons relatively more abundant in the meconium. The microbial compositions in the bovine amniotic fluid and meconium of the corresponding neonate did not show a significant correlation, contrary to earlier studies in humans (Collado et al., 2016; He et al., 2020).

471 Our analysis suggests that the bacterial DNA found in meconium does not necessarily originate

472 from the amniotic fluid. The maternal microbial components may be translocated via the 473 placental and umbilical blood vessels directly to the fetal internal organs. Microbial 474 macromolecules and even live bacteria may be transported by leukocytes via these blood 475 vessels (Perez et al., 2007).

476 A recent bovine study revealed differing microbial communities between the amniotic fluid 477 and the meconium (Guzman et al., 2020). As the fetus swallows the amniotic fluid, which is 478 concentrated and retained in the intestine as meconium, together with epithelial cells and 479 intestinal secretions, it is unexpected that the two would harbor completely different bacteria. However, the amniotic fluid microbiota may fluctuate dynamically over time, while meconium 
represents a more stable collection of substances accumulated over the gestation period.

484 chorion and amnion, rather than in the amniotic cavity (Bongso and Basrur, 1976).

485 The less permeable synepitheliochorial placenta evidenced by three distinct layers may restrict the translocation of bacteria and their components from the dam to the fetus in cattle, in

487 comparison to humans. The very low levels of bacterial DNA in the bovine amniotic fluid limit the sequencing depth and thus the precision of the compositional analyses. Our strict data decontamination protocol furthermore minimized the artefactual similarity arising from shared contaminants.

In conclusion, we found that the meconium of full-term calves delivered by elective caesarean section in most cases contains a small amount of diverse bacterial DNA and eventually

494 although rather rare, culturable bacteria. In the amniotic fluid, bacteria were not observed by 495 16S qPCR or culturing, but a microbial DNA profile was distinguishable from controls by 496 amplicon sequencing. Based on these results, bacterial components are translocated to the fetus 497 in utero, but the prenatal acquisition of live bacteria is likely not physiologically significant.

498 Data availability

499 The raw 16S rRNA gene amplicon sequencing dataset is fully available in the NCBI Sequence Read Archive (SRA) with the accession number PRJNA643145 after journal acceptance. 
501 Acknowledgements

502 We thank the owners of the cows for allowing sampling of their animals, and all clinicians and 503 graduate veterinary students for helping with the monitoring of the animals, performing the 504 surgery and sampling. Additionally, we thank Kirsi Lahti for expert technical assistance in the 505 laboratory.

\section{Author contributions}

507 MN, LL, AI and GO contributed to conception and design of the study. AH performed the

508 bioinformatics, statistical analyses and bacterial culture. AH and TG performed MALDI-TOF 509 analyses. LL and JG organized the sampling and collected the samples. AH and LL wrote the 510 manuscript. All authors contributed to manuscript preparation, read, and approved the 511 submitted version.

512

513 Ethics declaration

514 All experimental procedures were approved by the institutional ethics and animal welfare 515 committee of the Faculty of Veterinary Medicine (EC2018/002 - Ghent University, Belgium).

516 The cows' owners were informed about the study and gave their written consent for their cows' 517 participation. 


\section{References}

Aagaard, K., Ma, J., Antony, K. M., Ganu, R., Petrosino, J., and Versalovic, J. (2014). The placenta harbors a unique microbiome. Sci Transl Med 6, 237ra65. doi:10.1126/scitranslmed.3008599.

Al Alam, D., Danopoulos, S., Grubbs, B., Ali, N. A. B. M., MacAogain, M., Chotirmall, S. H., et al. (2020). Human Fetal Lungs Harbor a Microbiome Signature. Am. J. Respir. Crit. Care Med. doi:10.1164/rccm.201911-2127LE.

Alipour, M. J., Jalanka, J., Pessa-Morikawa, T., Kokkonen, T., Satokari, R., Hynönen, U., et al. (2018). The composition of the perinatal intestinal microbiota in cattle. Sci. Rep. 8, 10437. doi:10.1038/s41598-018-28733-y.

Andrews, S. (2010). FastQC: a quality control tool for high throughput sequence data. Available at: http://www.bioinformatics.babraham.ac.uk/projects/fastqc

Baker, J. M., Chase, D. M., and Herbst-Kralovetz, M. M. (2018). Uterine Microbiota: Residents, Tourists, or Invaders? Front. Immunol. 9. doi:10.3389/fimmu.2018.00208.

Bokulich, N. A., Kaehler, B. D., Rideout, J. R., Dillon, M., Bolyen, E., Knight, R., et al. (2018). Optimizing taxonomic classification of marker-gene amplicon sequences with QIIME 2's q2feature-classifier plugin. Microbiome 6, 90. doi:10.1186/s40168-018-0470-z.

Bolyen, E., Rideout, J. R., Dillon, M. R., Bokulich, N. A., Abnet, C. C., Al-Ghalith, G. A., et al. (2019). Reproducible, interactive, scalable and extensible microbiome data science using QIIME 2. Nat. Biotechnol. 37, 852-857. doi:10.1038/s41587-019-0209-9.

Bongso, T. A., and Basrur, P. K. (1976). Foetal fluids in cattle. Can. Vet. J. 17, 38.

Brunham, R. C., and Paavonen, J. (2020). Reproductive system infections in women: lower genital tract syndromes. Pathog. Dis. 78, 5.

Callahan, B. J., McMurdie, P. J., Rosen, M. J., Han, A. W., Johnson, A. J. A., and Holmes, S. P. (2016). DADA2: High-resolution sample inference from Illumina amplicon data. Nat. Methods 13, 581. doi:10.1038/nmeth.3869.

Cappelletti, M., Presicce, P., and Kallapur, S. G. (2020). Immunobiology of Acute Chorioamnionitis. Front. Immunol. 11. doi: 10.3389/fimmu.2020.00649.

Collado, M. C., Rautava, S., Aakko, J., Isolauri, E., and Salminen, S. (2016). Human gut colonisation may be initiated in utero by distinct microbial communities in the placenta and amniotic fluid. Sci Rep 6, 23129. doi:10.1038/srep23129.

de Goffau, M. C., Lager, S., Sovio, U., Gaccioli, F., Cook, E., Peacock, S. J., et al. (2019). Human placenta has no microbiome but can contain potential pathogens. Nature. doi:10.1038/s41586019-1451-5.

Dierikx, T. H., Visser, D. H., Benninga, M. A., van Kaam, A., de Boer, N. K. H., de Vries, R., et al. (2020). The influence of prenatal and intrapartum antibiotics on intestinal microbiota colonisation in infants: A systematic review. J. Infect. 
doi:https://doi.org/10.1016/j.jinf.2020.05.002.

Dornelles, L. V., Procianoy, R. S., Roesch, L. F., Corso, A. L., Dobbler, P. T., Mai, V., et al. (2020). Meconium microbiota predicts clinical early-onset neonatal sepsis in preterm neonates. $J$. Matern. Fetal Neonatal Med., 1-9.

Eisenhofer, R., Minich, J. J., Marotz, C., Cooper, A., Knight, R., and Weyrich, L. S. (2018). Contamination in Low Microbial Biomass Microbiome Studies: Issues and Recommendations. Trends Microbiol. doi:10.1016/j.tim.2018.11.003.

Ewels, P., Magnusson, M., Lundin, S., and Kaller, M. (2016). MultiQC: summarize analysis results for multiple tools and samples in a single report. Bioinformatics 32, 3047-8. doi:10.1093/bioinformatics/btw354.

Funkhouser, L. J., and Bordenstein, S. R. (2013). Mom knows best: the universality of maternal microbial transmission. PLoS Biol 11, e1001631. doi:10.1371/journal.pbio.1001631.

Glassing, A., Dowd, S. E., Galandiuk, S., Davis, B., and Chiodini, R. J. (2016). Inherent bacterial DNA contamination of extraction and sequencing reagents may affect interpretation of microbiota in low bacterial biomass samples. Gut Pathog. 8, 24.

Gomez de Agüero, M., Ganal-Vonarburg, S. C., Fuhrer, T., Rupp, S., Uchimura, Y., Li, H., et al. (2016). The maternal microbiota drives early postnatal innate immune development. Science 351, 1296. doi:10.1126/science.aad2571.

Gonçalves, L. F., Chaiworapongsa, T., and Romero, R. (2002). Intrauterine infection and prematurity. Ment. Retard. Dev. Disabil. Res. Rev. 8, 3-13.

Guzman, C.E., Wood, J.L., Egidi, E. et al. (2020) A pioneer calf foetus microbiome. Sci. Rep. 10, 17712.

Hansen, R., Scott, K. P., Khan, S., Martin, J. C., Berry, S. H., Stevenson, M., et al. (2015). First-pass meconium samples from healthy term vaginally-delivered neonates: an analysis of the microbiota. PloS One 10, e0133320.

Harrel, F. (2019). Hmisc: Harrell Miscellaneous. Available at: https://CRAN.Rproject.org/package $=$ Hmisc.

He, Q., Kwok, L. Y., Xi, X., Zhong, Z., Ma, T., Xu, H., ... \& Zhang, H. (2020). The meconium microbiota shares more features with the amniotic fluid microbiota than the maternal fecal and vaginal microbiota. Gut Microbes, 12(1), 1794266.

Hockney, R., Waring, G. J., Taylor, G., Cummings, S. P., Robson, S. C., Orr, C. H., et al. (2020). Fetal membrane bacterial load is increased in histologically confirmed inflammatory chorioamnionitis: A retrospective cohort study. Placenta 91, 43-51. doi:10.1016/j.placenta.2020.01.006.

Hofstad, T. (1992). "The genus Fusobacterium," in The prokaryotes (Springer), 4114-4126.

Husso, A., Jalanka, J., Alipour, M. J., Huhti, P., Kareskoski, M., Pessa-Morikawa, T., et al. (2020). The composition of the perinatal intestinal microbiota in horse. Sci. Rep. 10, 1-12. 
Jeon, S. J., Cunha, F., Vieira-Neto, A., Bicalho, R. C., Lima, S., Bicalho, M. L., et al. (2017). Blood as a route of transmission of uterine pathogens from the gut to the uterus in cows. Microbiome $5,1-13$.

Jimenez, E., Marin, M. L., Martin, R., Odriozola, J. M., Olivares, M., Xaus, J., et al. (2008). Is meconium from healthy newborns actually sterile? Res Microbiol 159, 187-93. doi:10.1016/j.resmic.2007.12.007.

Karstrup, C. C., Klitgaard, K., Jensen, T. K., Agerholm, J. S., and Pedersen, H. G. (2017). Presence of bacteria in the endometrium and placentomes of pregnant cows. Theriogenology 99, 41-47. doi:10.1016/j.theriogenology.2017.05.013.

Klein-Jöbstl, D., Quijada, N. M., Dzieciol, M., Feldbacher, B., Wagner, M., Drillich, M., et al. (2019). Microbiota of newborn calves and their mothers reveals possible transfer routes for newborn calves' gastrointestinal microbiota. PloS One 14(8): e0220554.

Kolkman, I., De Vliegher, S., Hoflack, G., Van Aert, M., Laureyns, J., Lips, D., et al. (2007). Protocol of the caesarean section as performed in daily bovine practice in Belgium. Reprod. Domest. Anim. 42, 583-589.

Korpela, K., and de Vos, W. M. (2018). Early life colonization of the human gut: microbes matter everywhere. Curr. Opin. Microbiol. 44, 70-78.

Krzyściak, W., Pluskwa, K. K., Jurczak, A., and Kościelniak, D. (2013). The pathogenicity of the Streptococcus genus. Eur. J. Clin. Microbiol. Infect. Dis. 32, 1361-1376.

Lagier, J.-C., Dubourg, G., Million, M., Cadoret, F., Bilen, M., Fenollar, F., et al. (2018). Culturing the human microbiota and culturomics. Nat. Rev. Microbiol. doi:10.1038/s41579-018-0041-0.

Lau, J. T., Whelan, F. J., Herath, I., Lee, C. H., Collins, S. M., Bercik, P., et al. (2016). Capturing the diversity of the human gut microbiota through culture-enriched molecular profiling. Genome Med 8, 72. doi:10.1186/s13073-016-0327-7.

Lauder, A. P., Roche, A. M., Sherrill-Mix, S., Bailey, A., Laughlin, A. L., Bittinger, K., et al. (2016). Comparison of placenta samples with contamination controls does not provide evidence for a distinct placenta microbiota. Microbiome 4, 29. doi:10.1186/s40168-016-0172-3.

Lisowska-Myjak, B., Skarżyńska, E., Wojdan, K., and Nasierowska-Guttmejer, A. (2019). Protein and peptide profiles in neonatal meconium. J. Obstet. Gynaecol. Res. 45, 556-564.

Liu, C.-J., Liang, X., Niu, Z.-Y., Jin, Q., Zeng, X.-Q., Wang, W.-X., et al. (2019). Is the delivery mode a critical factor for the microbial communities in the meconium? EBioMedicine 49, 354-363. doi:10.1016/j.ebiom.2019.10.045.

Lopez-Siles, M., Khan, T. M., Duncan, S. H., Harmsen, H. J., Garcia-Gil, L. J., and Flint, H. J. (2012). Cultured representatives of two major phylogroups of human colonic Faecalibacterium prausnitzii can utilize pectin, uronic acids, and host-derived substrates for growth. Appl Env. Microbiol 78, 420-428.

Lorenz, I., Fagan, J., and More, S. J. (2011). Calf health from birth to weaning. II. Management of diarrhoea in pre-weaned calves. Ir. Vet. J. 64, 9. 
Love, M. I., Huber, W., and Anders, S. (2014). Moderated estimation of fold change and dispersion for RNA-seq data with DESeq2. Genome Biol. 15, 550.

Malmuthuge, N., and Griebel, P. J. (2018). Fetal environment and fetal intestine are sterile during the third trimester of pregnancy. Vet. Immunol. Immunopathol. 204, 59-64.

Martin, M. (2011). Cutadapt removes adapter sequences from high-throughput sequencing reads. EMBnet.journal 17. doi:10.14806/ej.17.1.200.

Mayer, M., Abenthum, A., Matthes, J. M., Kleeberger, D., Ege, M. J., Hölzel, C., et al. (2012). Development and genetic influence of the rectal bacterial flora of newborn calves. Vet. Microbiol. 161, 179-185. doi:10.1016/j.vetmic.2012.07.023.

McMurdie, P. J., and Holmes, S. (2013). phyloseq: An R Package for Reproducible Interactive Analysis and Graphics of Microbiome Census Data. PLOS ONE 8, e61217. doi:10.1371/journal.pone.0061217.

Moore, R. L., Geraghty, A. A., Feehily, C., Saldova, R., Murphy, E. F., Van Sinderen, D., et al. (2020). Can a probiotic supplement in pregnancy result in transfer to the neonatal gut: A systematic review. Acta Obstet. Gynecol. Scand. doi:10.1111/aogs.13899.

Moore, S. G., Ericsson, A. C., Poock, S. E., Melendez, P., and Lucy, M. C. (2017). Hot topic: 16S rRNA gene sequencing reveals the microbiome of the virgin and pregnant bovine uterus. $J$. Dairy Sci. 100, 4953-4960.

Nadkarni, M. A., Martin, F. E., Jacques, N. A., and Hunter, N. \%J M. (2002). Determination of bacterial load by real-time PCR using a broad-range (universal) probe and primers set. 148, 257-266.

Oksanen J, F. G. B., Michael Friendly, Roeland Kindt, Pierre Legendre, Dan McGlinn, Peter R. Minchin, R. B. O’Hara, Gavin L. Simpson, Peter Solymos, M. Henry, H. Stevens, Eduard Szoecs and Helene Wagner (2019). vegan: Community Ecology Package. R package version 2.5-4. Available at: https://CRAN.R-project.org/package=vegan.

Olomu, I. N., Pena-Cortes, L. C., Long, R. A., Vyas, A., Krichevskiy, O., Luellwitz, R., et al. (2020). Elimination of "kitome" and "splashome" contamination results in lack of detection of a unique placental microbiome. BMC Microbiol. 20, 1-19.

Perez, P. F., Doré, J., Leclerc, M., Levenez, F., Benyacoub, J., Serrant, P., et al. (2007). Bacterial imprinting of the neonatal immune system: lessons from maternal cells? Pediatrics 119, e724-e732.

Perez-Munoz, M. E., Arrieta, M. C., Ramer-Tait, A. E., and Walter, J. (2017). A critical assessment of the "sterile womb" and "in utero colonization" hypotheses: implications for research on the pioneer infant microbiome. Microbiome 5, 48. doi:10.1186/s40168-017-0268-4.

Pipan, M. Z., Kajdič, L., Kalin, A., Plavec, T., and Zdovc, I. (2020). Do newborn puppies have their own microbiota at birth? Influence of type of birth on newborn puppy microbiota. Theriogenology. doi:10.1016/j.theriogenology.2020.04.014.

Quast, C., Pruesse, E., Yilmaz, P., Gerken, J., Schweer, T., Yarza, P., et al. (2013). The SILVA ribosomal RNA gene database project: improved data processing and web-based tools. Nucleic Acids Res 41, D590-6. doi:10.1093/nar/gks1219. 
R Studio Team (2020). RStudio: Integrated Development for R. RStudio Support. Available at: http://support.rstudio.com/hc/en-us/articles/206212048

Rackaityte, E., Halkias, J., Fukui, E. M., Mendoza, V. F., Hayzelden, C., Crawford, E. D., et al. (2020). Viable bacterial colonization is highly limited in the human intestine in utero. Nat. Med. 26, 599-607.

Rajaram, S., and Oono, Y. (2010). NeatMap-non-clustering heat map alternatives in R. BMC Bioinformatics 11, 1-9.

Reichhardt, M. P., Jarva, H., de Been, M., Rodriguez, J. M., Quintana, E. J., Loimaranta, V., et al. (2014). The salivary scavenger and agglutinin in early life: diverse roles in amniotic fluid and in the infant intestine. J. Immunol. 193, 5240-5248.

Reichhardt, M. P., and Meri, S. (2016). SALSA: a regulator of the early steps of complement activation on mucosal surfaces. Front. Immunol. 7, 85.

Reverdy, M. E., Freney, J., Fleurette, J., Coulet, M., Surgot, M., Marmet, D., et al. (1984). Nosocomial colonization and infection by Achromobacter xylosoxidans. J. Clin. Microbiol. $19,140-143$.

Rideout, J. R., Chase, J. H., Bolyen, E., Ackermann, G., Gonzalez, A., Knight, R., et al. (2016). Keemei: cloud-based validation of tabular bioinformatics file formats in Google Sheets. Gigascience 5, 27. doi:10.1186/s13742-016-0133-6.

Rihs, J. D., Brenner, D. J., Weaver, R. E., Steigerwalt, A. G., Hollis, D. G., and Yu, V. L. (1993). Roseomonas, a new genus associated with bacteremia and other human infections. J. Clin. Microbiol. 31, 3275-3283.

Romero, R., Gomez-Lopez, N., Winters, A. D., Jung, E., Shaman, M., Bieda, J., et al. (2019). Evidence that intra-amniotic infections are often the result of an ascending invasion-a molecular microbiological study. J. Perinat. Med. 47, 915-931.

Rossi, B., Merlo, B., Colleoni, S., Iacono, E., Tazzari, P. L., Ricci, F., et al. (2014). Isolation and in vitro characterization of bovine amniotic fluid derived stem cells at different trimesters of pregnancy. Stem Cell Rev. Rep. 10, 712-724.

Savini, V., Catavitello, C., Masciarelli, G., Astolfi, D., Balbinot, A., Bianco, A., et al. (2010). Drug sensitivity and clinical impact of members of the genus Kocuria. J. Med. Microbiol. 59, 1395-1402.

Scholz, C. F., and Kilian, M. (2016). The natural history of cutaneous propionibacteria, and reclassification of selected species within the genus Propionibacterium to the proposed novel genera Acidipropionibacterium gen. nov., Cutibacterium gen. nov. and Pseudopropionibacterium gen. nov. Int. J. Syst. Evol. Microbiol. 66, 4422-4432.

Seferovic, M. D., Pace, R. M., Carroll, M., Belfort, B., Major, A. M., Chu, D. M., et al. (2019). Visualization of microbes by $16 \mathrm{~S}$ in situ hybridization in term and preterm placentas without intraamniotic infection. Am. J. Obstet. Gynecol. 221, 146.e1-146.e23. doi:10.1016/j.ajog.2019.04.036. 
Segata, N., Izard, J., Waldron, L., Gevers, D., Miropolsky, L., Garrett, W. S., et al. (2011). Metagenomic biomarker discovery and explanation. Genome Biol. 12, 1-18.

Stinson, L. F., Boyce, M. C., Payne, M. S., and Keelan, J. A. (2019). The not-so-sterile womb: evidence that the human fetus is exposed to bacteria prior to birth. Front. Microbiol. 10, 1124.

Stinson, L. F., Keelan, J. A., and Payne, M. S. (2018). Comparison of Meconium DNA Extraction Methods for Use in Microbiome Studies. Front Microbiol 9, 270. doi:10.3389/fmicb.2018.00270.

Theis, K. R., Romero, R., Greenberg, J. M., Winters, A. D., Garcia-Flores, V., Motomura, K., et al. (2020a). No Consistent Evidence for Microbiota in Murine Placental and Fetal Tissues. mSphere 5, e00933-19. doi:10.1128/mSphere.00933-19.

Theis, K. R., Romero, R., Winters, A. D., Jobe, A. H., and Gomez-Lopez, N. (2020b). Lack of evidence for microbiota in the placental and fetal tissues of rhesus macaques. mSphere 5. doi: 10.1128/mSphere.00210-20.

Thornton Philip, K. (2010). Livestock Production: Recent Trends, Future Prospects. Nairobi Kenya, doi: 10.1098/rstb. 2010.0134 Phil. Trans R Soc B 27 Sept. 2010 Vol 365 No 155428532867.

Touchon, M., Cury, J., Yoon, E.-J., Krizova, L., Cerqueira, G. C., Murphy, C., et al. (2014). The genomic diversification of the whole Acinetobacter genus: origins, mechanisms, and consequences. Genome Biol. Evol. 6, 2866-2882.

Vandenplas, Y., Carnielli, V. P., Ksiazyk, J., Luna, M. S., Migacheva, N., Mosselmans, J. M., et al. (2020). Factors affecting early-life intestinal microbiota development. Nutrition, 110812.

Yeoman, C. J., Ishaq, S. L., Bichi, E., Olivo, S. K., Lowe, J., and Aldridge, B. M. (2018). Biogeographical Differences in the Influence of Maternal Microbial Sources on the Early Successional Development of the Bovine Neonatal Gastrointestinal tract. Sci Rep 8, 3197. 